\title{
Impact of Throughput in Enhancing the Efficiency of Cognitive Radio Ad Hoc Network - A Study
}

\author{
V. Jayaraj, Associate Profess or \\ School of Computer Science and Engineering, Bharathidas an University, Tamilnadu, India \\ E-mail: jaya_v@yahoo.com \\ J. Jegathesh Amalraj, Research Scholar \\ School of Computer Science and Engineering, Bharathidas an University, Tamilnadu, India \\ E-mail:amal.jas@gmail.com \\ L. Nagarajan, Research Scholar \\ School of Computer Science and Engineering, Bharathidas an University, Tamilnadu, India \\ E-mail: mcadirector@gmail.com
}

\begin{abstract}
Cognitive Radio Ad Hoc Networks (CRAHNs) constitute a viable solution to solve the current problems of inefficiency in the spectrum allocation, and to deploy highly reconfigurable and selforganizing wireless networks. Cognitive Radio (CR) devices are envisaged to utilize the spectrum in an opportunistic way by dynamically accessing different licensed portions of the spectrum. However the phenomena of channel fading and primary cum secondary interference in cognitive rad io networks does not guarantee application demands to be achieved continuously over time. Availability of limited spectrum and the inadequate spectrum resource usage necessitates a new communication standard to utilize the existing wireless spectrum opportunistically. This paper discusses currently existing mechanism for providing better efficiency by utilizing cognitive network intelligence. The frequencies used are utilized to the maximum extent without any interference. This paper aims in comparing the techniques used for enhancing the throughput in CR.
\end{abstract}

Index Terms - Adhoc network, Spectrum Sensing, Spectrum Decision block, PU Aware policy, Throughput

\section{Introduction}

An Adhoc Network is a collection of wireless mobile hosts forming a temporary network without the aid of any established infrastructure or centralized administration ${ }^{[7]}$. Each node in adhoc wireless network in similar in features. It is necessary to introduce some intelligence in the adhoc networks in order to improve their throughput efficiency. Cognitive Radio approach is used to provide the necessary intelligence. CR enabled devices are intelligent enough and can listen to the surrounding wireless environment and can select the appropriate frequency band, modulation scheme or specific power level automatically ${ }^{[8]}$. By Cognitive intelligence, an ability of self decision making can be incorporated in the wireless adhoc networks.

The frequency spectrum is a limited resource for wireless communications and gets congested owing to the need to accommodate the diverse types of air interface used in next generation wireless networks. This spectrum if utilized in an efficient manner, can lead to better utilization of the network.

Wireless technology has enabled the development of increasingly diverse applications and devices resulting in an exponential growth in its usage and services. These advancements have made the radio frequency spectrum a scarce resource, and consequently, its efficient use is of the ultimate importance. To cope with the growing demand, network design focused on increasing the spectral efficiency by making use of advancement in Cognitive Radio technology. Cognitive Radio can reduce the spectrum shortage problem by enabling unlicensed users equipped with Cognitive Radios to reuse and share the licensed spectrum bands ${ }^{[3]}$. Cognitive Radio is capable of sensing the environmental conditions and automatically adapt its operating parameters in order to enhance network performance. A Cognitive Radio has a cognitive process that can perceive current network and environmental conditions, and then plan, decide and act on those conditions. The $\mathrm{CR}$ can learn from these adaptations and use them to make future decisions such as changing transmission parameters dynamically, all while taking into account end-to-end goals. Cognitive Radio technology is the key technology that enables a CRAHN to use spectrum in a dynamic manner ${ }^{[9]}$. 
The paper is organized as follows: The Section II gives an introduction about the Cognitive Radio (CR). The Section III describes the existing techniques in CR for enhancing the throughput maximization and Section IV compares the results and interpretations of the existing techniques and Section $\mathrm{V}$ concludes the result.

\section{Cognitive Radio}

A Cognitive Radio is a kind of two-way radio that automatically changes its transmission or reception parameters, in a way where the entire wireless communication network of which it is a node communicates efficiently, while avoiding interference with licensed or licensed exempt users. This alteration of parameters is based on the active monitoring of several factors in the external and internal radio environment, such as radio frequency spectrum, user behavior and network state. The Fig. 1 shows a sample CR network.

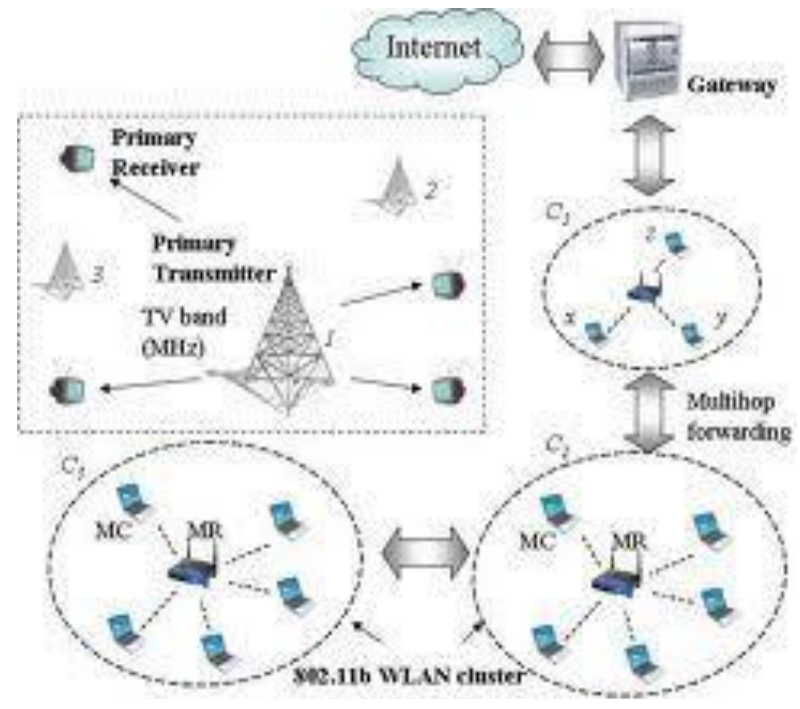

Fig. 1: A Sample CR Network

A "Cognitive Radio" is a radio that can change its transmitter parameters based on interaction with the environment in which it operates.

From this definition, two main characteristics of the Cognitive radio can be defined as follows ${ }^{[9]}$ :

- Cognitive capability: Cognitive capability refers to the ability of the radio technology to capture or sense the information from its radio environment. This capability cannot simply be realized by monitoring the power in some frequency bands of interest but more sophisticated techniques, such as autonomous learning and action decision are required in order to capture the temporal and spatial variations in the radio environment and avoid interference to other users. Through this capability, the portions of the spectrum that are unused at a specific time or location can be identified. Consequently, the best spectrum and appropriate operating parameters can be selected.
- Re-configurability: The cognitive capability provides spectrum awareness whereas re-configurability enables the radio to be dynamically programmed according to the radio environment. More specifically, the cognitive radio can be programmed to transmit and receive on a variety of frequencies and to use different transmission access technologies supported by its hardware design.

According to the network architecture, cognitive radio (CR) networks can be classified as the infrastructure-based CR network and the CRAHNs. The infrastructure-based CR network has a central network entity such as a base station in cellular networks or an access point in wire less local area networks (LANs). On the other hand, the CRAHN does not have any infrastructure backbone. Thus, a CR user can communicate with other CR users through ad hoc connection on both licensed and unlicensed spectrum bands.

Regulatory bodies in various countries (including the Federal Communications Commission in the United States, and Ofcom in the United Kingdom) found that most of the radio frequency spectrum was inefficiently utilized. For example, cellular network bands are overloaded in most parts of the world, but many other frequency bands, such as military, amateur radio and paging frequencies are not. Independent studies performed in some countries confirmed that observation, and concluded that spectrum utilization depends strongly on time and place ${ }^{[4]}$. Moreover, fixed spectrum allocation prevents rarely used frequencies (those assigned to specific services) from being used by unlicensed users, even when their transmissions would not interfere at all with the assigned service. This was the reason for allowing unlicensed users to utilize licensed bands whenever it would not cause any interference (by avoiding them whenever legitimate user presence is sensed). This paradigm for wire less communication is known as Cognitive Radio.

Since the spectrum present is limited, all users cannot be allowed to access this network. Hence users are divided into two basic categories.

1. Primary Users: Since the spectrum present is limited, all users cannot be allowed to access this network of any other unlicensed users. Primary users do not need any modification or additional functions for coexistence.

2. Secondary Users or Unlicensed Users: They access the licensed spectrum as a visitor, by opportunistically transmitting on the spectrum holes.

\section{Comparison of the Existing Techniques in CR for Throughput Maximization}

Throughput is defined as the average rate of successful message delivery over a communication 
channel. It is measured in terms of number of bits passing through network in one second and it is measured in bits per second.

\subsection{CR implementation by using Switch}

The Fig 3 shows the typical transmitter and receiver diagram for $802.11 \mathrm{a}$ WLAN system. ${ }^{[1]}$ aimed to enhance the efficiency of throughput in the 802.11a WLAN system. Relation between channel capacity and the throughput performance is shown below ${ }^{[2]}$.

\section{$\mathrm{C}=\mathrm{BW} * \log _{2}(1=\mathrm{SNR}) \mathrm{bits} / \mathrm{sec}$}

BW represents bandwidth of the operation in $\mathrm{Hz}$ and SNR indicates the signal to noise ratio of the channel. To enhance the throughput efficiency, the bandwidth operation is increased by using CR concept. This is because CR allows the flexible use of spectrum in wireless environment.

The output of the receiver in the form of 0 feedbacks is used in the control element. A switch is proposed to insert at the output of the transmitter. The switch will have an input and 3 output units 1,2 and 3. 1 and 3 units are for data input and 2 is for the control input.

When the input level of the 2 is equal to or greater than the set threshold, then the input 1 of the switch will be in active. In case of input 2 has a level less than the set value of the threshold mean input 3 will be in active. This helps in controlling the transmitted power level based on the feedback. So the secondary user will transmit in lower power level without causing interference to the primary. The secondary user can transmit at higher power when the input 2 is in the lower level. Thus the throughput performance of the channels will be enhanced. The proposed switch is shown in Fig 2.

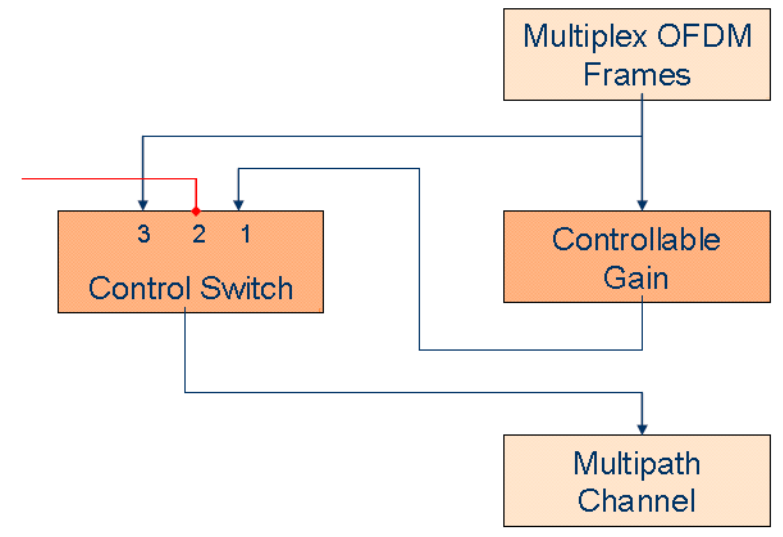

Fig. 2: The Insertion of Control Element to obtain output power control in IEEE 802.11 a Link

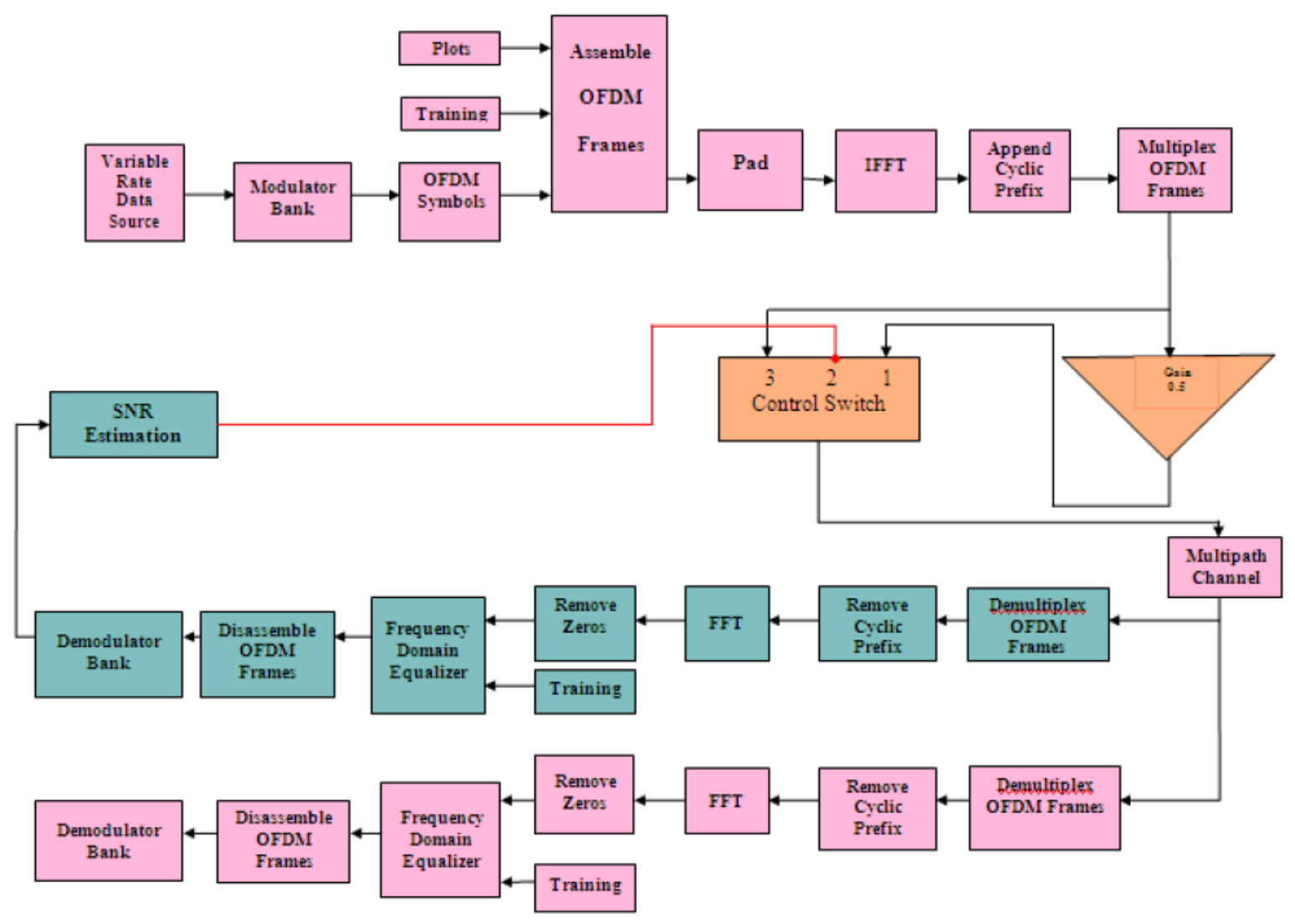

Fig. 4: Proposed Improvement in the System Model 


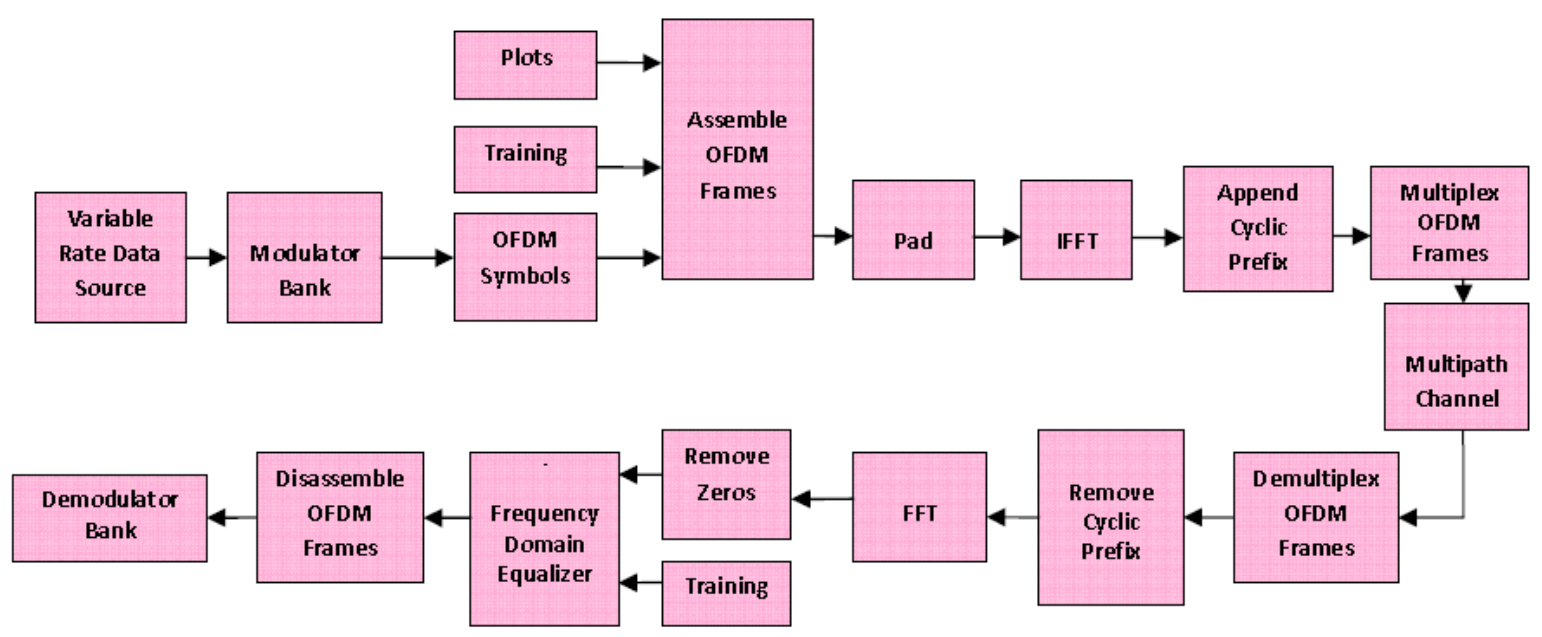

Fig. 3: 802.11a WLAN System Transmitter and Receiver Diagram ${ }^{[1]}$

\subsection{PU Aware Allocation Policy}

The CR user activity is modeled by using the cognitive cycle model described in Fig. 5. Here we depict the three components of the cognitive cycle model: the spectrum sensing block, the spectrum mobility block ${ }^{[5]}$ and the spectrum decision block. Each CR alternately senses the channel and transmits data. If the spectrum sensing block detects a PU in the specific portion of the spectrum in use, then the CR user should vacate the spectrum and continue its communication in another portion of the spectrum. The handoff and protocol-reconfiguration are performed by the spectrum mobility block. Also, the CR must select a new channel to use based on its QoS requirements. The spectrum decision block is responsible for the channel selection. Otherwise, if the current channel is found free from PU activity, then the CR user may transmit on it, by using MAC layer coordination schemes which prevent collisions with other CR users using an overlapping portion of the spectrum.

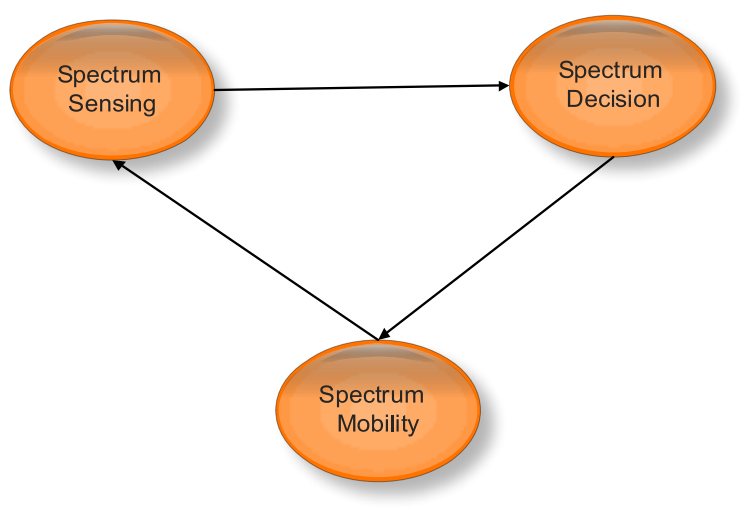

Fig. 5: System Architecture representing the Cognitive cycle ${ }^{[4]}$

To perform these processes, the system is provided with log files that maintain the details about user activity and channel details.

\section{PU activity log file format}

The PU-log file contains information about (i) PUs location and characteristics and (ii) PUs activity over time. The first part of the file is composed of entries with this format:

$$
\langle\text { id, } x, y, \text { channel, tpower }\rangle
$$

where id is the unique identifier of a PU, x, y are its location, channel and tpower are the channel and transmitting power used by the PU, respectively.

The second part of the file describes the activity of each PU over time, by means of a list of entries with this format:

$$
\text { 〈id, arrivaltime, departuretime〉 }
$$

where id is the PU identifier, arrival time is the simulation time when the PU enters the ON period and starts transmitting, and departure time is the simulation time when the PU enters the OFF period and ceases transmitting.

\section{Channel-log file format}

The channel-log file contains information about (i) physical channel characteristics and (ii) channel quality. The spectrum data module is responsible for loading the information from the file and making them available to the other modules. For each CR channel, the log file contains an entry with this format

\section{$\langle$ id, frequency, bandwidth, noise $\rangle$}

where id is the identifier of the channel (a number between 1 and $\mathrm{N}$ ), frequency is the channel central frequency, bandwidth is the raw bandwidth of the channel (e.g. $11 \mathrm{Mb} / \mathrm{s}$ ), and noise is the average value of the noise on that channel. By using bandwidth and noise, 
and by knowing the power received at a given location, it is possible to model the average BER experienced by the receiver node, for the QPSK modulation.

\section{Spectrum Sensing Block}

Channel sensing is modeled as a lookup function on the PU-log file, for the current channel (say channel i), sensing interval ts and simulation time t. The readings from the file are then adjusted on the basis of the sensing accuracy, so that the final outcome of the sensing process is a binary response whether a PU signal is detected or not on the current channel.

More in details, a CR user performing sensing on a channel $i$ at time $t$ checks the PU-log file if there is an entry for a PU (say P) satisfying these two conditions (C):

o C1: $\mathrm{P}$ is transmitting on the same channel $\mathrm{i}$, or on an adjacent channel, for the time interval $[\mathrm{t} ; \mathrm{t}+\mathrm{ts}]$;

o C2: The amount of power injected on a channel $i$ by node $\mathrm{P}$ and received by node $\mathrm{C}$ (i.e. $\mathrm{PrC}$ ) is higher than the sensitivity threshold PthC:

$$
\text { Pr C } \geq \text { PthC }
$$

Signal propagation is modeled through a generalized free-space model, i.e. PrC is computed by node $\mathrm{C}$ as follows:

$$
\operatorname{PrC}=[\mathrm{Pt} \cdot \mathrm{Ct} /(\mathrm{d} \alpha)] \mathrm{k}
$$

where $\mathrm{Pt}$ is the transmitting power of $\mathrm{P}$, the constant Ct captures different transmission properties such as the antenna gains and height, $\alpha$ is the attenuation factor, $d$ is the physical distance between $\mathrm{P}$ and $\mathrm{C}$, and $\mathrm{k}$ is the overlap factor between channel $i$ and the central channel frequency used by P. We consider the overlap factors as $1,0.5$ and 0.25 for 0,1 and 2 channel spacings from the PU's central channel frequency, respectively. Overlap factors can be adjusted to accommodate for different systems and spectral overlap mask characteristics. If both conditions $\mathrm{C} 1$ and $\mathrm{C} 2$ are verified, then the variable puON is set as true. However, this is not enough to guarantee a correct PU detection, because node $\mathrm{C}$ might misdetect the presence of $\mathrm{P}$. In the current implementation, we use a simplified equation for $\mathrm{Pd}$, as a function of the sensing time interval (ts) only. A PU signal is detected if puON is set as true, with probability $\mathrm{Pd}$. In this case, a notification is sent to the spectrum decision block.

\section{Spectrum Decision Block}

The spectrum decision block is responsible for (i) deciding the spectrum policy and (ii) choosing the next channel to be used by CR users, in case a PU activity detection requires a channel switch. Each time a PU activity is detected on the current channel, a CR user can adopt two different policies:

- Switch policy: The CR user immediately vacates the current channel ${ }^{[6]}$ and moves to another portion of the frequency spectrum.

- Stay and Wait policy: The CR user does not vacate the current channel, but stops transmitting on it, because it must not interfere with the PU activity. In this case, a notification is sent to the sensing block. As soon as the PU activity ceases, the CR user restarts its operations on the current channel.

In the case of channel switch, the CR user must find another portion of the spectrum for its operations. The channel selection can be performed at the link-layer (by the spectrum decision block) or at the routing layer ${ }^{[10]}$. In the case of channel selection performed by the spectrum decision block, three channel allocation schemes can be used:

1. Random Allocation: The CR user chooses randomly among the available $\mathrm{N}$ channels.

2. Sequential Allocation: The CR user visits all the $\mathrm{N}$ channels, by a round-robin algorithm:

$$
\text { next_channel }=(\text { current_channel }+1) \% \mathrm{~N}
$$

3. PU-aware Allocation: The CR user chooses the channel providing the highest transmission opportunities, by considering the amount of interference injected on each channel by each PU and by neighbor CR users.

\section{Spectrum Mobility Block}

The spectrum mobility block is invoked when the spectrum decision block decides that the CR user mus $t$ vacate the current channel. It receives from the spectrum decision block the new channel to switch to (e.g. next_channel). The delay induced by the channel switch is modeled by using a timer. A CR user is not allowed to utilize the radio interface for co mmunication during the handoff operation. When the handoff process is completed, the spectrum sensing block is invoked to detect the PU activity on next_channel. If next_channel is found free of PU activities, then a spectrum handoff notification is sent to the upper layer, and protocol reconfiguration is performed at the network layer.

\section{Results and Discussions}

The Fig 6 depicts the variations in SNR and bit rate. The proposed system shows stability in both the cases. The Fig 7 and Fig 8 show the system response for the two cases shown. 

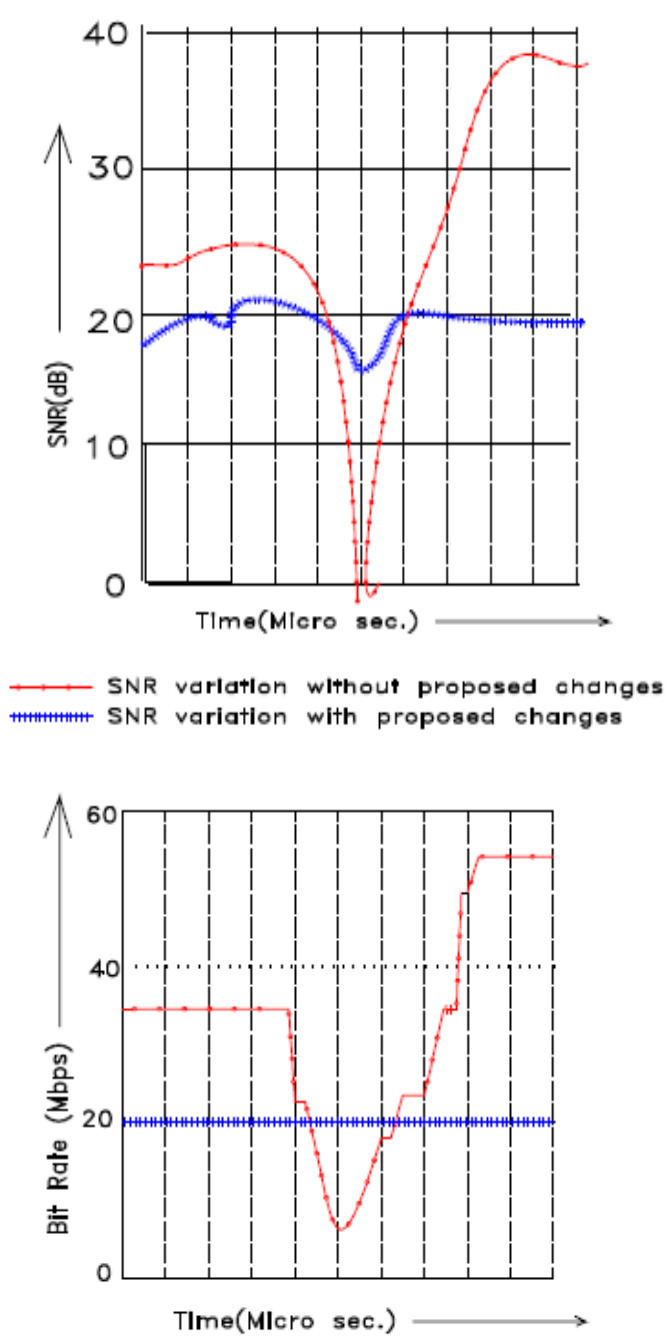

Bit Rate variation without proposed changes Bit Rate variation with proposed changes

Fig. 6: SNR and bit rate Variations with and without proposed system [1]

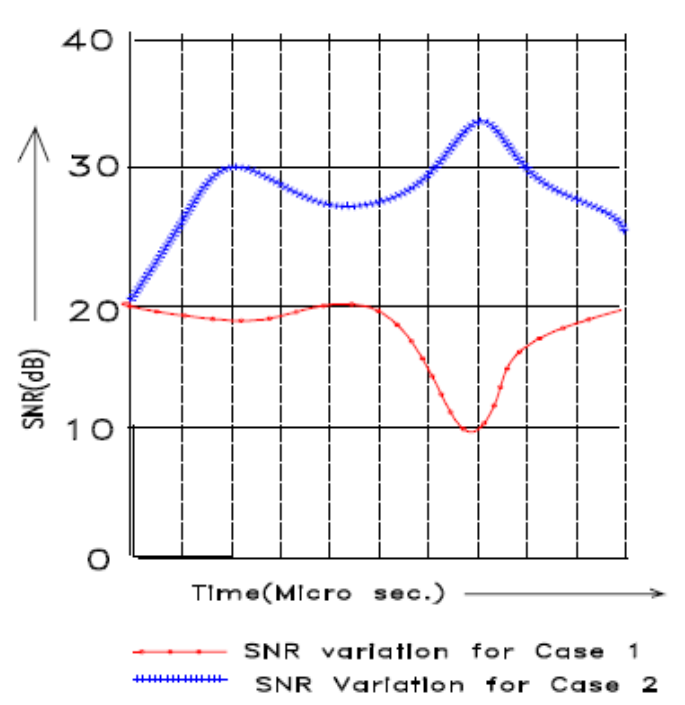

Fig. 7: SNR variations transmitted signal in case 1 when channel is occupied and case 2 when channel is empty ${ }^{[1]}$
If channel is preoccupied it transmits with preagreed lower signal as in Fig 7 and in full power, when the channel is empty as in Fig 8.

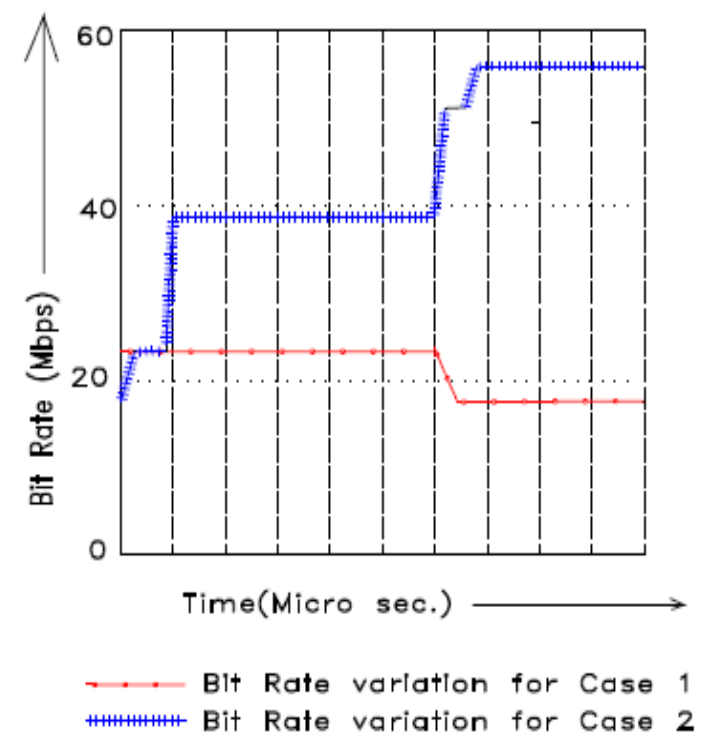

Fig. 8: Bit rate variations in case 1 when channel 1 is occupied and case 2 when channel is empty

The proposed system is close to the concept of CSMA/CA at MAC layer of WLAN. But in this concept secondary user need not stop the transmissions and no need to change the frequency or channel of transmission as in CSMA/CA.

The PU Aware Policy is simulated by using Network Simulator (NS2), with nodes of varying intensity and capacity links. Better transmissions and lesser switching of channels are observed. Interference is reduced and both Secondary User and Primary user had faster channel acquisition time. The Fig 9 shows the less switching of channels by using PU Aware policy.

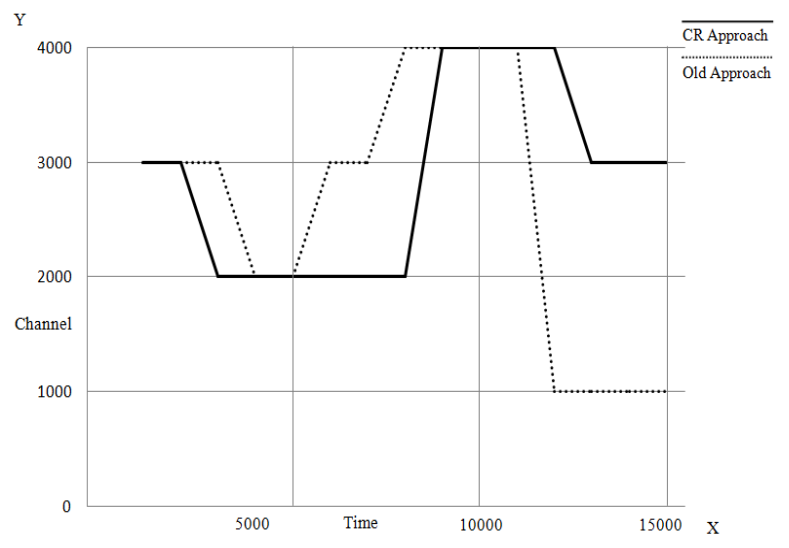

Fig. 9: Comparison of Channel Switching using PU Aware Policy ${ }^{[4]}$

The Fig 10 shows the less degree of interference observed because of using the PU aware policy for CR. The graph is obtained by taking number of users in $\mathrm{X}$ axis and amount of interference in the $\mathrm{Y}$ axis. 


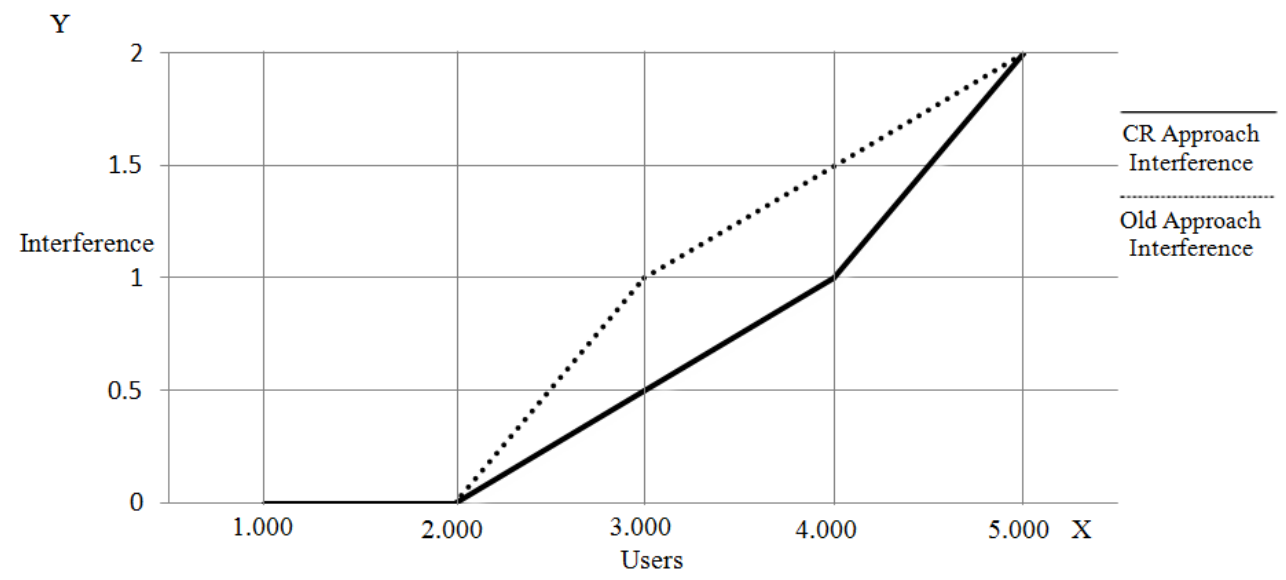

Fig. 10: Comparison of Interference

\section{Conclusion}

The underlay approach of transmission of signals is used in most of the case in CR. In the overlay approach the secondary users can transmit only in the spectrum holes. While comparing the switch and PU aware policy, the later one is found to be better in implementation. Inclusion of switch in a circuit is a cost and time oriented factor, which wastes most of the resources. So PU Aware policy is found to be the best one since it allows the secondary users to transmit without any interference and changes can be made with less effort and cost. Switching of channels by using the PU Aware policy is also ought to enhance the throughput which is the future enhancement to be carried.

\section{References}

[1] Charushila A xay Patel, Sanjay Kumar, "Enhancing Throughput Efficiency of Adhoc Wireless Networks using Cognitive Radio Approach", International Conference on Devices and Communications, IEEE, 2011, 978-1-244-9190$2 / 11$.

[2] Taub \& Schiling, Principles of Communication System, TMH, 2004.

[3] Ismai Butun, Cagatay, Talay, Turgay, Altilar, Murad, Khalid, Ravi Sankar, "Impact of Mobility Prediction on the Performance of Cognitive Radio Networks", Wireless Telecommunications Symposium, IEEE, 2010, 978-1-4244-6557-6/10.

[4] J.Jegathesh Amalraj and Dr. V.Jayaraj, "Efficient Spectrum Sharing and Allocation Schemes for Throughput Enhancements in a Cognitive Radio Network", International Conference on Computing, Communication and Networking Technology (ICCCNT'12), IEEE, 2012, Paper No: IEEE20180.

[5] $\mathrm{Xu}$ Ling, Song Li, "Enhancing the capacity of Spectrum sharing Cognitive Radio Networks",
IEEE Transactions on Vehicular Technology, IEEE, 2011, Volume 60, No.8, 0018-9545.

[6] Cuiran Li, Chengshu Li, "Oppurtunistic Spectrum Access in Cognitive Radio Networks", International Joint Conference on Neural Networks, IEEE, 2008, 978-1-4244-1821-3/08.

[7] Pravin Ghosekar, Girish Katkar and Dr. Pradip Ghorpade, "Mobile Ad Hoc Networking: Imperatives and Challenges", 2010, International Journal of Computer Applications .

[8] Patel C.A, "Enhancing Throughput Efficiency of Adhoc Wireless Networks Using Cognitive Radio Approach", International Conference on Devices and Communications, IEEE, 2011, 978-1-42449189-6.

[9] Ian F. Akyildiz, Won-Yoel Lee, Kaushik R.Chowdhury, "CRAHNs: Cognitive Radio Ad Hoc Networks", 2007, Elsevier, Journal Ad Hoc Networks.

[10] Cuiran Li, Chengshu Li, "Opportunistic Spectrum Access in Cognitive Radio Networks", International Joint Conference on Neural Networks, IEEE, 2008, 978-1-4244-1821-3/08.

\section{Authors' Profile}

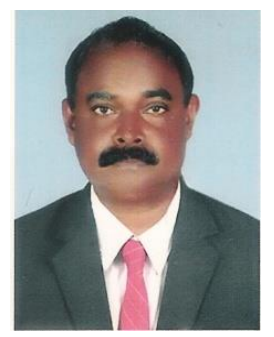

Dr .V. Jayaraj

Dr. V.Jayaraj holds a Ph.D in Computer Science from Bharathidasan University, Tiruchirappalli, Tamilnadu. He has more than 20 years of teaching experience and has guided more than 50 M.Phil scholars. He is currently working as an Associate Professor in Computer Science and guiding research scholars in Bharathidasan University. Presently he is doing his research on Mobile Computing and Data Mining domains. 


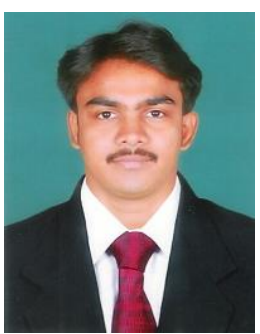

\section{J. Jegathesh Amalraj}

J.Jegathesh Amalraj received his Master Degree in Computer Applications from St.Joseph's College, Tiruchirappalli, Tamilnadu, India in 2011. He is currently doing his Doctoral Degree in School of Computer Science and Engineering, Bharathidasan University, Tamilnadu and his research works are under the domains of Wireless and Ad Hoc Networks.

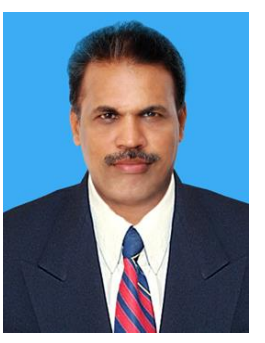

\section{L.Nagarajan}

L. Nagarajan working as a Professor and Head, in the department of Computer Science and Applications, Adaikalamtha College, Thanjavur, Tamilnadu. And he is having more than 25 years of experience in Teaching and Industry. Currently he is doing his Doctoral Degree in School of Computer Science and Engineering, Bharathidasan University, Tiruchirappalli, Tamilnadu and his research works are under the domains of Wireless Ad-Hoc Networks.

How to cite this paper: V. Jayarai, J. Jegathesh Amalrai, L. Nagarajan,"Impact of Throughput in Enhancing the Efficiency of Cognitive Radio Ad Hoc Network - A Study", International Journal of Information Technology and Computer Science(IJITCS), vol.5. no.10, pp.70-77, 2013. DOI: 10.5815/ijitcs.2013.10.07 